\title{
On the Stability of a Power Control Algorithm for Wireless Networks in the presence of Time-Varying Delays
}

\author{
Themistoklis Charalambous* and Yassine Ariba**
}

\begin{abstract}
This paper studies the robustness of the well known Foschini-Miljanic power control algorithm with respect to time-varying delays. Since delays are omnipresent in wireless networks, this problem is of practical importance. It has been proven in the past that no matter how large the delays are, the Foschini-Miljanic algorithm still converges. However, this was based on the assumption that the delays are constant over time, which is not always met in practice. Therefore, the problem addressed in this paper is how the algorithm behaves under the time-varying delays case. Firstly, we provide the conditions under which the system is stable by means of a Linear Matrix Inequality (LMI) and using a semi-definite optimization solver we show the validity of our results through illustrative examples.
\end{abstract}

\section{INTRODUCTION}

In wireless networks where Quality of Service (QoS) is affected by interference and wireless nodes are being supplied by batteries with limited lifetime, power control has become an attractive research area (e.g. [1], [2], [3], [4], [5], [6], [7]). On one hand, the wireless channel is a shared, interference-limited medium and transmission power of nodes need to be adjusted so that it is high enough to reach the intended receiver. On the other hand, the transmitter is powered by a finite energy source and hence a power control scheme which is energy-efficient is required that causes minimal interference at other nodes.

The authors in [2] proposed a power control algorithm, the now well known as the Foschini-Miljanic (FM) algorithm, that provides for distributed on-line power control of wireless networks with user-specific Signal-to-Interferenceand-Noise-Ratio (SINR) requirements. Furthermore, this algorithm yields the minimum transmitter powers that satisfy these requirements. A vast number of publications have extended this work to account for additional issues, such as constrained power [3] and admission control [8]. However, it was only recently that time-delays were taken into account for this algorithm [9]. In [9], it is proven that if the FoschiniMiljanic algorithm converges when there are no time-delays present in the network then it is guaranteed to converge in the presence of delays, as well. However, this work relies on the assumption that the delays are constant over time, which is not always met in practice.

In general, the notions concerning the stability of time delay systems can be classified into two categories. Namely, delay-independent stability (e.g. [10]) and delay-dependent

\footnotetext{
* Control Laboratory, Department of Engineering, University of Cambridge, UK. tc257@eng. cam.ac.uk

** LAAS-CNRS, Université de Toulouse, 7, avenue du Colonel Roche, F-31077 Toulouse, France. $\{$ yariba\} 01 aas. fr
}

stability (e.g. [11]), depending on whether they contain the delay argument as a parameter. As pointed out in the literature, the delay-independent stability conditions may be restrictive when the delays are comparatively small. In many practical problems, the delays are time-varying and/or only known to be bounded, without any extra information. Hence, the stability analysis of time-varying delays in networks has become more significant than that with constant time-delays.

But, why is the study of time-varying delays in wireless networks so important? In wireless networks, especially ad hoc networks, distributed algorithms necessitate communication among nodes for updating. Hence, delays are inevitably introduced into the network for various reasons (processing time (coding and decoding), propagation delays and availability of the channel for transmission are the most prominent), which by their nature are not constant throughout the operation of the network. Therefore, time-varying delays are omnipresent in such networks and stability analysis of distributed algorithms for such a realistic condition is necessary.

In this work, we extend the work done in [9] by investigating the robustness of the Foschini-Miljanic algorithm in the case where the network experiences time-varying delays. At first, we derive the stability conditions for which the system is stable by proposing a Lyapunov-Krasovskii functional in the form of a Linear Matrix Inequality (LMI) [12]. It is an effective and practical methodology providing LMI conditions which can be solved efficiently with semidefinite optimization solvers in a polynomial time ensuring the global stability of the wireless network. The validity of our result is indicated through numerical examples, showing that the Foschini-Miljanic power control algorithm is able to converge to the optimal vector of powers even in cases of time-varying delays, when the nodes adjust their proportionality constants $\left(k_{i}\right)$ accordingly. The channel gain matrix considered here is time-invariant. Therefore, the proposed stability conditions are just valid for the time-invariant (or slowly time-varying) channels and do not consider the general time-varying case.

The rest of the paper is organized as follows. In Section II, the system model is described and some preliminary results are presented, giving a brief review of the Foschini-Miljanic algorithm and relevant results. In Section III the conditions for stability of the Foschini-Miljanic algorithm with timevarying delays are derived while in section IV numerical examples supporting the theoretical results are presented. At last, in Section $\mathrm{V}$ conclusions are drawn and directions for future work are given. 


\section{Notations And Preliminaries}

\section{A. Notations}

For two symmetric matrices $A$ and $B, A \succ B$ means that $A-B$ is positive definite and $A \succeq B$ means that $A-B$ is semi-positive definite. $A^{T}$ and $A^{-1}$ denote the transpose and inverse of matrix $A$ respectively. $|A|$ is the element-wise absolute value of the matrix (i.e. $|A| \triangleq\left[\left|A_{i j}\right|\right]$ ), $A \leq B$ is the element-wise inequality between matrices $\mathrm{A}$ and $\mathrm{B}$ and $A<B$ is the strict element-wise inequality between $\mathrm{A}$ and B. A nonnegative matrix (i.e. a matrix whose elements are nonnegative) is denoted by $A \geq 0$ and a positive matrix is denoted by $A>0 . \sigma(A)$ denotes the spectrum of matrix $A, \lambda(A)$ denotes an element of the spectrum of matrix $A$, and $\rho(A)$ denotes its spectral radius. $\operatorname{det}(A)$ denotes the determinant of matrix $A$ and $\operatorname{diag}\left(x_{i}\right)$ the matrix with elements $x_{1}, x_{2}, \ldots$ on the leading diagonal and zeros elsewhere.

\section{B. System model}

We consider a planar network where the links are assumed to be unidirectional and each node is supported by an omnidirectional antenna. This can be represented by a graph $\mathscr{G}=(\mathscr{N}, \mathscr{L})$, where $\mathscr{N}$ is the set of all nodes and $\mathscr{L}$ is the set of the active links in the network. Each node can be a receiver or a transmitter only at each time instant due to the half-duplex nature of the wireless transceiver. Each transmitter aims to communicate with a single node (receiver) only, which cannot receive from more than one nodes simultaneously. We denote by $\mathscr{T}$ the set of transmitters and $\mathscr{R}$ the set of receivers in the network.

The channel gain on the link between transmitter $i$ and receiver $j$ is denoted by $g_{i j}$ and incorporates the mean path-loss as a function of distance, shadowing and fading, as well as cross-correlations between signature sequences. All the $g_{i j}$ 's are positive and can take values in the range $(0,1]$. The power level chosen by transmitter $i$ is denoted by $p_{i}$ and the intended receiver is also indexed by $i . v$ denotes the variance of thermal noise at the receiver, which is assumed to be additive Gaussian noise. The link quality is measured by the Signal-to-Interference-and-Noise-Ratio (SINR). The interference power at the $i^{\text {th }}$ receiver, $I_{i}$, includes the interference from all the transmitters in the network (apart from the communicating transmitter) and the thermal noise, and is given by

$$
I_{i}=\sum_{j \neq i, j \in \mathscr{T}} g_{j i} p_{j}+v .
$$

Therefore, the SINR at the receiver $i, \Gamma_{i}$, is given by

$$
\Gamma_{i}=\frac{g_{i i} p_{i}}{\sum_{j \neq i, j \in \mathscr{T}} g_{j i} p_{j}+v} .
$$

Due to the nature of the wireless channel, it is necessary to ensure Quality of Service (QoS) at the wireless links in terms of SINR in wireless networks. Independently of nodal distribution and traffic pattern, a transmission from transmitter $i$ to its corresponding receiver is considered successful if the SINR of the receiver is greater or equal to $\gamma_{i}\left(\Gamma_{i} \geq \gamma_{i}\right)$, called the capture ratio and it is dependent on the modulation and coding characteristics of the radio [13]. Therefore we require,

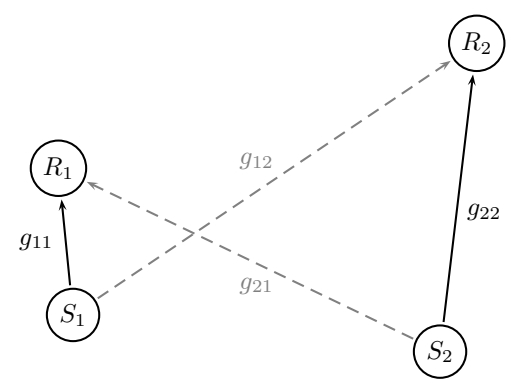

Fig. 1. An example of a network consisting of two communication pairs only. Each pair $i$ consists of a transmitter $S_{i}$ and a receiver $R_{i}$ connected with a solid line while the grey dotted arrows indicate the interference that transmitters cause to the neighboring receivers.

$$
\frac{g_{i i} p_{i}}{\sum_{j \neq i, j \in \mathscr{T}} g_{j i} p_{j}+v} \geq \gamma_{i}
$$

\section{Preliminary results}

Equation (3) after manipulation, is equivalent to the following

$$
p_{i} \geq \gamma_{i}\left(\sum_{j \neq i, j \in \mathscr{T}} \frac{g_{j i}}{g_{i i}} p_{j}+\frac{v}{g_{i i}}\right) .
$$

In matrix form, for a network consisting of $n$ communication pairs, this can be written as

$$
\mathbf{p} \geq \Gamma G \mathbf{p}+\eta
$$

where

$$
\begin{aligned}
& \Gamma=\operatorname{diag}\left(\gamma_{i}\right) \\
& \mathbf{p}=\left(\begin{array}{llll}
p_{1} & p_{2} & \ldots & p_{n}
\end{array}\right)^{T} \\
& G_{i j}= \begin{cases}0 & , \text { if } i=j, \\
\frac{g_{j i}}{g_{i i}} & , \text { if } i \neq j .\end{cases} \\
& \eta_{i}=\frac{\gamma_{i} v}{g_{i i}}
\end{aligned}
$$

Let,

$$
C=\Gamma G
$$

so that (5) can be written as

$$
(I-C) \mathbf{p} \geq \eta
$$

The matrix $C$ has nonnegative elements and it is reasonable to assume that is irreducible, since we are not considering totally isolated groups of links that do not interact with each other. By the Perron-Frobenius theorem [14], we have that the spectral radius of the matrix $C$ is a simple eigenvalue, while the corresponding eigenvector is positive componentwise. The necessary and sufficient condition for the existence of a nonnegative solution to inequality (7) for every positive vector $\eta$ is that $(I-C)^{-1}$ exists and is nonnegative. However, $(I-C)^{-1} \geq 0$ if and only if $\rho(C)<1$ [15] (Theorem 2.5.3), [16] . 
Therefore, the necessary and sufficient condition for (7) to have a positive solution $\mathbf{p}^{*}$ for a positive vector $\eta$ is that the Perron-Frobenius eigenvalue of the matrix $C$ is less than 1. That is, there exists a set of powers such that all the senders can transmit simultaneously and still meet their QoS requirements (minimum SINR for successful reception).

In this work, it is assumed that there exist separate channels that enable the receivers to send their interference measurements to their respective transmitters. Therefore, we consider a set of communication pairs in a specific channel, such that each pair consists of a transmitter and a receiver at all times, without reversing the communication direction.

The Foschini-Miljanic algorithm, [2], succeeds in attaining the required SINRs for all nodes in the network if a solution exists and fails if there does not exist a solution. The following differential equation is defined in [2] in order to model the continuous-time power dynamics:

$$
\frac{d p_{i}(t)}{d t}=k_{i}\left(-p_{i}(t)+\gamma_{i}\left(\sum_{j \neq i, j \in \mathscr{T}} \frac{g_{j i}}{g_{i i}} p_{j}(t)+\frac{v}{g_{i i}}\right)\right)
$$

where $k_{i} \in \mathbb{R}, k_{i}>0$, denotes the proportionality constant, $g_{j i}$ denotes the channel gain on the link between transmitter $j$ and receiver $i$ and $\gamma_{i}$ denotes the desired SINR. It is assumed that each transmitter $i$ has knowledge of the interference at its receiver only (1).

In matrix form this is written as

$$
\dot{\mathbf{p}}(t)=-K H \mathbf{p}(t)+K \eta
$$

where $K=\operatorname{diag}\left(k_{i}\right)$ and

$$
H_{i j}= \begin{cases}1 & , \text { if } i=j \\ -\gamma_{i} \frac{g_{j i}}{g_{i i}} & , \text { if } i \neq j\end{cases}
$$

For this differential equation, it is proved [2] that the system will converge to the optimal set of solutions, $\mathbf{p}^{*}>0$, for any initial power vector, $\mathbf{p}(0)>0$. Therefore, the distributed algorithm (8) for each communication pair, leads to global stability of the system.

It is proven in [9] that, if the system is globally stable when there are no delays into the network, then it is also stable for arbitrarily large time-delays, $T_{i}>0$, and for any proportionality constant, $k_{i}>0$. However, as aforementioned, this work relies on the assumption that the delays are constant over time, which in hardly ever met in practice.

\section{MAIn RESUlts}

Since the transmitter uses information (interference) provided by the receiver, unavoidably, there exists a time-delay on the information used while updating the power (i.e. delays from receiver to transmitter). However, this delay is rarely kept constant during the operation of the network. Consequently, for a more realistic representation of the algorithm we introduce time-varying delays $\left(T_{i}(t)\right)$ to the FoschiniMiljanic algorithm and analyze the stability conditions for the system. Therefore, the differential equation (8) becomes

$$
\dot{p}_{i}(t)=k_{i}\left(-p_{i}(t)+\gamma_{i}\left(\sum_{j \neq i, j \in \mathscr{T}} \frac{g_{j i}}{g_{i i}} p_{j}\left(t-T_{i}(t)\right)+\frac{v}{g_{i i}}\right)\right) \text {. }
$$

In matrix form, in the case of $N$ nodes, (10) can be written as

$$
\dot{\mathbf{p}}(t)=-K \mathbf{p}(t)+K\left(\sum_{k=1}^{N} A_{d_{k}} \mathbf{p}\left(t-T_{k}(t)\right)+\eta\right)
$$

where

$$
\begin{aligned}
\mathbf{p}(t) & =\left(\begin{array}{c}
p_{1}(t) \\
\vdots \\
p_{N}(t)
\end{array}\right) \\
A_{d_{k_{i j}}} & = \begin{cases}0 & , \text { if } j=k \text { or } i \neq k, \\
\gamma_{k} \frac{g_{j i}}{g_{k k}} & , \text { otherwise. }\end{cases} \\
K & =\operatorname{diag}\left(k_{i}\right), \\
\eta & =\left(\begin{array}{c}
\gamma_{1 \frac{v}{g_{11}}} \\
\vdots \\
\gamma_{N} \frac{v}{g_{N N}}
\end{array}\right) .
\end{aligned}
$$

Therefore, the stability of (11) is a equivalent to and can be assessed by the study of the following system:

$$
\dot{\mathbf{p}}(t)=-K \mathbf{p}(t)+K\left(\sum_{k=1}^{N} A_{d_{k}} \mathbf{p}\left(t-T_{k}(t)\right)\right)
$$

In the constant delay case, preliminary result [9] has guaranteed the stability of the Foschini-Miljanic algorithm using the multi-variate Nyquist criterion [17]. This latter study established conditions on $k_{i}$ 's such that the algorithm is stable independently from delays, i.e. whatever the values of delays $T_{i}$. However, this approach appears to be unrealistic since the delays in practice are usually time-varying.

The aim of this work is the establishment of a stability condition which takes into account that delays are time-varying and hence, it comprises a more realistic representation of the Foschini-Miljanic Algorithm. To this end, a Lyapunov-Krasovskii method (see for example [18]) is utilized, which is an extension of the traditional Lyapunov theory. It is an effective and practical methodology providing LMI conditions which can be solved efficiently with semi-definite optimization solvers in a polynomial time.

Assume that the delays $\left(T_{i}(t)\right.$ for all $\left.i \in \mathscr{T}\right)$ are continuously differentiable, bounded $\left(0 \leq T_{i}(t) \leq T_{i_{m}}, T_{i_{m}}>0\right)$, and $\dot{T}_{i}(t) \leq \alpha_{i}$, $\alpha_{i} \geq 0, \forall t$ and $\forall i \in \mathscr{T}$. The following theorem states the conditions for which the FM algorithm subjected to time-varying delays is stable.

Theorem 1: Given scalars $T_{i_{m}}>0$ and $\alpha_{i} \geq 0, \forall i \in \mathscr{T}$, the system (12) is asymptotically stable for any time-varying delays $T_{i}(t)$ satisfying $0 \leq T_{i}(t) \leq T_{i_{m}}$, and $\dot{T}_{i}(t) \leq \alpha_{i}, \forall t$ and $\forall i \in \mathscr{T}$, if there exists $N \times N$ matrices $Q_{k} \succ 0, S_{k} \succ 0, R_{k} \succ 0, k=\{1, \ldots, N\}$ and $N \times N$ diagonal matrices $Z \succ 0, X \succ 0, Y \succ 0$ such that the following LMI holds:

$$
\left[\begin{array}{ll}
\Theta_{11} & \Theta_{12} \\
\Theta_{21} & \Theta_{22}
\end{array}\right] \prec 0 \text { and } \mathrm{Z} \succeq \mathrm{W}
$$


where

$$
\begin{aligned}
& \Theta_{11}=\left[\begin{array}{cccc}
T & \frac{1}{T_{1 m}} R_{1}+X A_{d_{1}} & \ldots & \frac{1}{T_{N_{m}}} R_{N}+X A_{d N} \\
\frac{1}{T_{1_{m}}} R_{1}+A_{d_{1}}^{T} X & U_{1} & 0 & \ldots \\
\vdots & \vdots & \ddots & 0 \\
\frac{1}{T_{N_{m}}} R_{N}+A_{d_{N}}^{T} X & 0 & \ldots & U_{N}
\end{array}\right] \\
& \Theta_{12}=\left[\begin{array}{cccc}
0 & \cdots & 0 & -Y \\
\frac{1}{T_{1 m}} R_{1} & 0 & \vdots & A_{d 1}^{T} Y \\
\vdots & \ddots & \vdots & \vdots \\
0 & \cdots & \frac{1}{T_{N_{m}}} R_{N} & A_{d N}^{T} Z
\end{array}\right], \\
& \Theta_{21}=\Theta_{12}^{T} \text {, } \\
& \Theta_{22}=\left[\begin{array}{cccc}
V_{1} & \cdots & 0 & 0 \\
\vdots & \ddots & \vdots & \vdots \\
0 & \cdots & V_{N} & 0 \\
0 & \cdots & 0 & -Z
\end{array}\right] \\
& \text { and } \\
& T=\sum_{k=1}^{N}\left[Q_{k}+S_{k}-\frac{1}{T_{k_{m}}} R_{k}\right]-2 X, \\
& U_{k}=-\left(1-\alpha_{k}\right) Q_{k}-\frac{2}{T_{k_{m}}} R_{k} \text {, } \\
& V_{k}=-S_{k}-\frac{1}{T_{k_{m}}} R_{k}, \\
& W=\sum_{k=1}^{N} T_{k_{m}} R_{k} .
\end{aligned}
$$

The stabilizing gain $K=\operatorname{diag}\left(k_{i}\right)$ is given by $K=Z^{-1} Y$.

Proof: Consider the following Lyapunov-Krasovskii functional [18], extended for the multiple time-varying delays case:

$$
\begin{aligned}
V\left(t, \mathbf{p}_{t}\right) & =\mathbf{p}^{T}(t) P \mathbf{p}(t)+\sum_{k=1}^{N} \int_{t-T_{k}(t)}^{t} \mathbf{p}^{T}(\theta) Q_{k} \mathbf{p}(\theta) d \boldsymbol{\theta} \\
& +\sum_{k=1}^{N} \int_{t-T_{k_{m}}}^{t} \mathbf{p}^{T}(\theta) S_{k} \mathbf{p}(\theta) d \boldsymbol{\theta}+\sum_{k=1}^{N} \int_{t-T_{k_{m}}}^{t} \int_{\theta}^{t} \dot{\mathbf{p}}^{T}(u) R_{k} \dot{\mathbf{p}}(u) d u d \theta
\end{aligned}
$$

Given that $Q_{k}, S_{k}, R_{k}$ for $k \in \mathscr{T}$ are positive-definite matrices and $P$ is a diagonal positive matrix, we can conclude that for some $\varepsilon>0$, the Lyapunov-Krasovskii functional condition $V\left(\mathbf{p}_{t}\right) \geq \varepsilon\left\|\mathbf{p}_{t}(0)\right\|$ is satisfied [18]. The derivative along the trajectories of (12) leads to

$$
\begin{aligned}
\dot{V}\left(t, \mathbf{p}_{t}\right)= & 2 \mathbf{p}^{T}(t) P \dot{\mathbf{p}}(t) \\
& +\sum_{k=1}^{N}\left[\mathbf{p}^{T}(t) Q_{k} \mathbf{p}(t)\right. \\
& \left.-\left(1-\dot{T}_{k}(t)\right) \mathbf{p}^{T}\left(t-T_{k}\right) Q_{k} \mathbf{p}\left(t-T_{k}\right)\right] \\
& +\sum_{k=1}^{N}\left[\mathbf{p}^{T}(t) S_{k} \mathbf{p}(t)-\mathbf{p}^{T}\left(t-T_{k_{m}}\right) S_{k} \mathbf{p}\left(t-T_{k_{m}}\right)\right] \\
& +\sum_{k=1}^{N}\left[T_{k_{m}} \dot{\mathbf{p}}^{T}(t) R_{k} \dot{\mathbf{p}}(t)-\int_{t-T_{k_{m}}}^{t} \dot{\mathbf{p}}^{T}(\theta) R_{k} \dot{\mathbf{p}}(\theta) d \theta\right] .
\end{aligned}
$$

Consider the integral term:

$$
\begin{aligned}
\int_{t-T_{k_{m}}}^{t} \dot{\mathbf{p}}^{T}(\theta) R_{k} \dot{\mathbf{p}}(\theta) d \theta= & \int_{t-T_{k_{m}}}^{t-T_{k}(t)} \dot{\mathbf{p}}^{T}(\theta) R_{k} \dot{\mathbf{p}}(\theta) d \theta \\
& +\int_{t-T_{k}(t)}^{t} \dot{\mathbf{p}}^{T}(\theta) R_{k} \dot{\mathbf{p}}(\theta) d \theta
\end{aligned}
$$

and applying the Jensen's inequality [18] to both terms, we have

$$
\begin{gathered}
-\int_{t-T_{k}(t)}^{t} \dot{\mathbf{p}}^{T}(\theta) R_{k} \dot{\mathbf{p}}(\theta) d \theta \leq-v_{k}^{\prime}(t) R_{k} v_{k}(t), \\
-\int_{t-T_{k_{m}}}^{t-T_{k}(t)} \dot{\mathbf{p}}^{T}(\theta) R_{k} \dot{\mathbf{p}}(\theta) d \theta \leq-w_{k}^{\prime}(t) R_{k} w_{k}(t),
\end{gathered}
$$

where $v_{k}(t)=\left(\mathbf{p}(t)-\mathbf{p}\left(t-T_{k}(t)\right)\right)$ and $w_{k}(t)=(\mathbf{p}(t-$ $\left.T_{k}(t)\right)-\mathbf{p}\left(t-T_{k_{m}}\right)$. Combining (16) and (17), we obtain:

$$
\dot{V}\left(t, \mathbf{p}_{t}\right) \leq \xi^{T}(t) \Gamma \xi(t)+\dot{\mathbf{p}}^{T}(t) W \dot{\mathbf{p}}(t),
$$

where

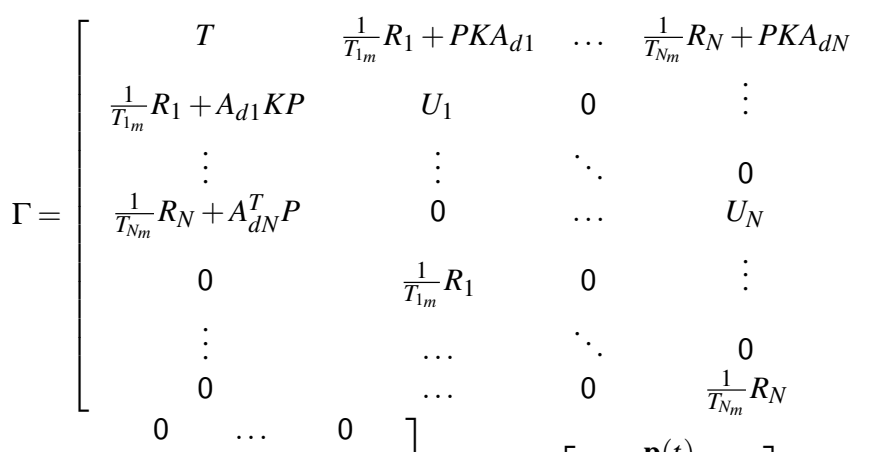

$$
\begin{aligned}
& \left.\begin{array}{ccc}
0 & \cdots & 0 \\
\frac{1}{T_{1_{m}}} R_{1} & 0 & \vdots \\
\vdots & \ddots & \vdots \\
0 & \cdots & \frac{1}{T_{N_{m}}} R_{N} \\
V_{1} & \cdots & 0 \\
\vdots & \ddots & \vdots \\
0 & \cdots & V_{N}
\end{array}\right], \quad \xi(t)=\left[\begin{array}{c}
\mathbf{p}(t) \\
\mathbf{p}\left(t-T_{1}(t)\right) \\
\vdots \\
\mathbf{p}\left(t-T_{N}(t)\right) \\
\mathbf{p}\left(t-T_{1_{m}}\right) \\
\vdots \\
\mathbf{p}\left(t-T_{N_{m}}\right)
\end{array}\right]
\end{aligned}
$$

and $T=\sum_{k=1}^{N}\left[Q_{k}+S_{k}-\frac{1}{T_{k_{k}}} R_{k}\right]-K P-P K, U_{k}=-(1-$ $\left.\alpha_{k}\right) Q_{k}-\frac{2}{T_{k_{m}}} R_{k}, V_{k}=-S_{k}-\frac{1}{T_{k_{m}}} R_{k}$ and $W=\sum_{k=1}^{N} T_{k_{m}} R_{k} . \alpha_{k}$ is the upper bound on $\dot{T}_{k}(t)$. If we introduce a diagonal positive definite matrix $Z$ (as decision variable) such that $Z \succeq W$, the following condition implies $\dot{V}(\mathbf{p}) \leq 0$ :

$$
\xi^{T}(t) \Gamma \xi(t)+\dot{\mathbf{p}}^{T}(t) Z \dot{\mathbf{p}}(t)<0 .
$$

The condition can be re-written as

$$
\xi^{T}(t) \Gamma \xi(t)+\xi^{T}(t) N^{T} Z N \xi(t)<0
$$

which is equivalent to

$$
\begin{aligned}
& \Gamma+N^{T} Z N \prec 0 \text { and } \mathrm{Z} \succeq \mathrm{W} \\
\Leftrightarrow & {\left[\begin{array}{cc}
\Gamma & N^{T} Z \\
Z N & -Z
\end{array}\right] \prec 0 \text { and } \mathrm{Z}-\mathrm{W} \succeq 0, }
\end{aligned}
$$


with $N=\left[\begin{array}{lllllll}-K & K A_{d 1} & \ldots & K A_{d N} & 0 & \ldots & 0\end{array}\right]$. Then, we perform the change of variable: $Y=Z K$ and $X=P K$. Since $P$ and $Z$ are diagonal positive definite matrices and $K$ is also a diagonal matrix with positive components (that is, the proportionality constants $k_{i}$ are positive - a necessary condition as introduced in [2]) then $Y$ and $X$ are required to be diagonal positive matrices. This latter additional condition on $X$ is necessary to ensure the existence of a diagonal positive matrix $P=X K^{-1}$. Thus the condition (21) is expressed as condition (13).

Theorem 1 provides a practical and systematic condition that provides $k_{i}$ 's ensuring the stability of the FM algorithm. Once these gains are embedded in each node (via broadcasting or by communication with a central controller), each power $p_{i}(t)$ used by the transmitter $i$ will converge towards its corresponding equilibrium point $p_{i_{0}}$ according to (11) in a distributed manner, where $p_{i_{0}}=\gamma_{i}\left(\sum_{j \neq i, j \in \mathscr{T}} \frac{g_{j i}}{g_{i i}} p_{j_{0}}+\frac{v}{g_{i i}}\right)$ is the minimal power required to satisfy the SINR constraints.

\section{NUMERICAL EXAMPLES}

This section aims to elucidate the proposed methodology via illustrative examples. Firstly, consider an ad-hoc network consisting of 4 communicating pairs, i.e. 8 mobile devices in total. For this example we set the SINR threshold and the thermal noise for each node to $\gamma_{i}=3$ and $v=0.04$ Watts, respectively. The initial power $p_{i}(0)$ for all transmitters is set to 1 Watt. The network is described by matrix $C_{1}-$ which is obtained by (6) - and it is schematically shown in Figure 2 .

$$
C_{1}=\left[\begin{array}{cccc}
0 & 0.0163 & 0.0108 & 0.0212 \\
0.0250 & 0 & 1.5124 & 0.2566 \\
0.0213 & 0.2146 & 0 & 0.3564 \\
0.0771 & 0.0111 & 0.1224 & 0
\end{array}\right]
$$

Applying Theorem 1 to this representative example, a

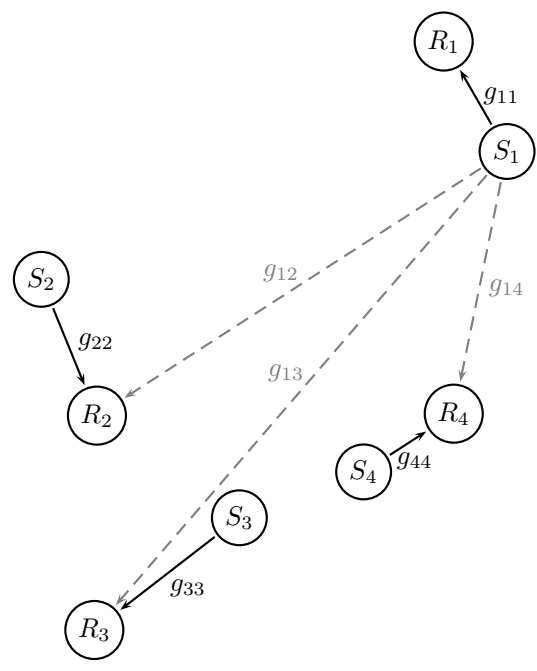

Fig. 2. Example of a wireless ad-hoc network of $n=8$ nodes, consisting of four communication pairs $\left\{S_{i} \rightarrow R_{i}\right\}$. The grey dotted arrows are included to indicatively show the interference caused to the receivers by $S_{1}$. stabilizing gain matrix $\left(K_{1}\right)$ is obtained,

$$
K_{1}=\operatorname{diag}(0.1280,0.0608,0.0859,0.0919) \text {. }
$$

The power adjustment by each transmitter is simulated and the results are depicted in Figure 3.

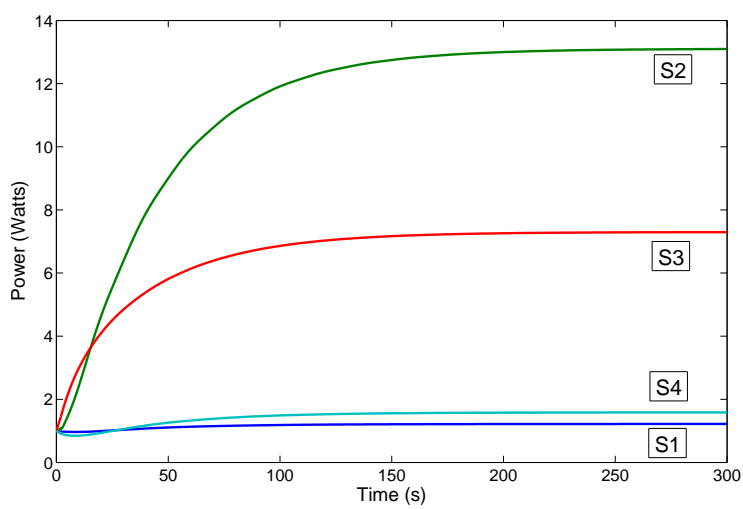

Fig. 3. Simulation of the network represented in Figure 2 Power levels converge to the desired SINR and to the optimal power vector.

The communication time-varying delays between the different pairs have been simulated with different signal generators (sine and sawtooth with different frequencies) such that $\dot{T}_{k} \leq \alpha_{k}=1$. The maximal values of each delays are $T_{m}=\{2,3,4,2\}$ where each $T_{k_{m}}$ is expressed in $s$ (seconds).

Consider a second example of a wireless network with 6 communicating pairs (see Figure 4 ) characterized by matrix (23).

$C_{2}=\left[\begin{array}{cccccc}0 & 0.0414 & 0.2074 & 0.2925 & 0.3998 & 0.1345 \\ 0.0159 & 0 & 0.0506 & 0.0043 & 0.0422 & 1.164 \\ 0.7335 & 0.0626 & 0 & 0.0364 & 0.0477 & 0.4231 \\ 0.6359 & 0.0222 & 0.0644 & 0 & 0.3283 & 0.0447 \\ 0.0227 & 0.0536 & 0.0155 & 0.0215 & 0 & 0.0407 \\ 0.0228 & 0.1114 & 0.2458 & 0.0030 & 0.011 & 0\end{array}\right]$
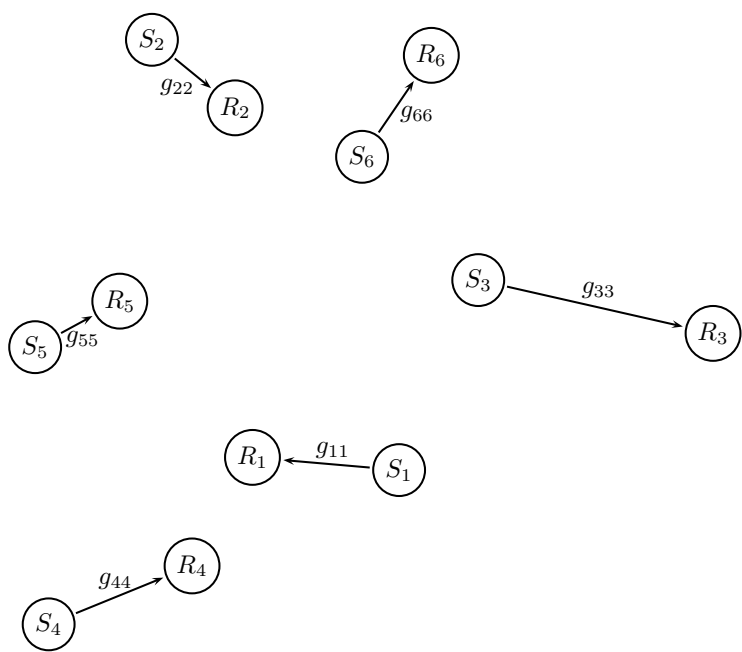

Fig. 4. Example of a wireless ad-hoc network of $n=12$ nodes, consisting of six communication pairs $\left\{S_{i} \rightarrow R_{i}\right\}$. Interference caused is not depicted in the figure. 
Setting $\gamma_{i}$ and $v$ as previously and considering time-varying delays such that $\dot{T}_{k} \leq \alpha_{k}=1$ and $T_{m}=\{4,2,8,4,2,3\}(s)$, the stability condition (13) provides a stabilizing gain matrix $K_{2}$

$$
K_{2}=\operatorname{diag}(0.0300,0.0435,0.0401,0.0427,0.0538,0.0391) .
$$

Then simulation has been performed and the evolution of the power used by each transmitter is shown in Figure 5.

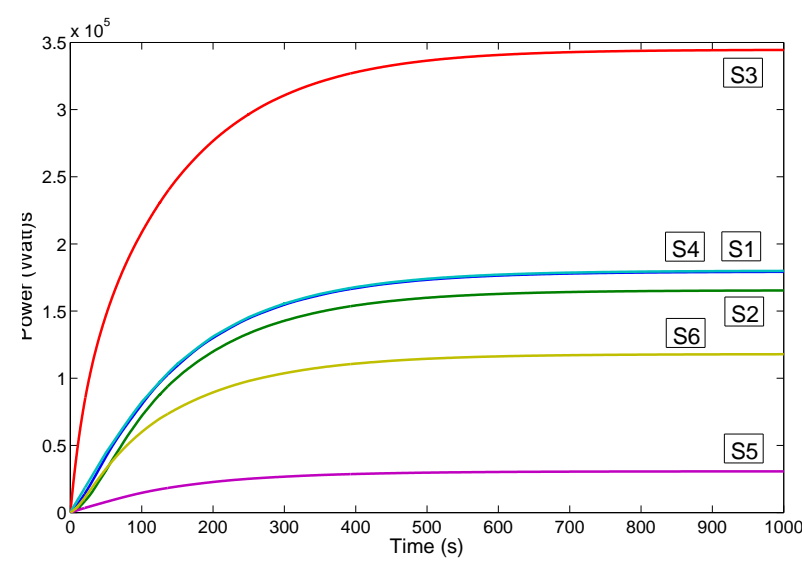

Fig. 5. Simulation of the network represented in Figure 4 Power levels converge to the desired SINR and to the minimal power vector

The significance of the result can be appreciated especially in cases where a central controller is able to obtain an estimate on the upper bounds of the maximum delays that transmitters may experience, calculate appropriate gains that guarantee global stability of the system and disseminate the information required to the corresponding users. Then, the system will operate in a distributed fashion. This is easily applicable in cellular networks where a base station can be considered as the central controller that can carry out the calculations and broadcast the information to the corresponding transmitters.

\section{CONCLUSIONS AND Future WORK}

\section{A. Conclusions}

In this paper, the delay-dependent stability analysis of the time-varying delay Foschini-Miljanic algorithm has been studied by means of a Lyapunov-Krasovskii functional. A LMI is derived which provides a practical and systematic condition for stability of the Foschini-Miljanic algorithm. The LMI condition is centrally solved with the aid of semidefinite LMI solvers in polynomial time and the proportionality constants $k_{i}$ are fed back to the transmitters. Once the corresponding gains are embedded to each transmitter, the network operates in a distributed manner and it is asymptotically stable.

\section{B. Future Work}

Extension to this work is the derivation of upper bounds on the proportionality constants $k_{i}$ if necessary, such that the algorithm operates in a distributed way without the need for any information dissemination by a central station or controller to the rest of the nodes in the network.
It would also be interesting to investigate in an analytical way and obtain the relationship between the convergence rate of the algorithm with the magnitude of the time delay. If the convergence time grows much faster than the communication delays, then the algorithm will not operate well in practice under the presence of large time delays.

\section{REFERENCES}

[1] J. Zander, "Distributed co-channel interference control in cellular radio systems," IEEE Transaction on Vehicular Technology, vol. 41, no. 3, pp. 305-311, August 1992.

[2] G. Foschini and Z. Miljanic, "A Simple Distributed Autonomous Power Control Algorithm and its Convergence," IEEE Transactions on Vehicular Technology, vol. 42, no. 4, pp. 641-646, November 1993.

[3] S. Grandhi, J. Zander, and R. Yates, "Constrained power control," Wireless Personal Communications, vol. 2, no. 3, August 1995.

[4] R. D. Yates, "A framework for uplink power control in cellular radio systems," IEEE Journal on Selected Areas in Communications, vol. 13, pp. 1341-1347, September 1995.

[5] T. ElBatt and A. Ephremides, "Joint Scheduling and Power Control for Wireless Ad-hoc Networks," in Proceedings of IEEE INFOCOM, 2002.

[6] Z. Gajic, D. Skataric, and S. Koskie, "Optimal SIR-based Power Updates in Wireless CDMA Communication Systems," in IEEE Conference on Decision and Control, vol. 5, December 2004, pp. 5146-5151.

[7] M. Chiang, C. W. Tan, D. P. Palomar, D. O'Neill, and D. Julian, "Power Control by Geometric Programming," IEEE Transactions on Wireless Communications, vol. 6, no. 7, pp. 2640-2651, July 2007.

[8] N. Bambos, S. C. Chen, and G. J. Pottie, "Channel Access Algorithms with Active Link Protection for Wireless Communication Networks with Power Control," IEEE/ACM Transactions on Networking, vol. 8, no. 5 , pp. 583-597, 2000

[9] T. Charalambous, I. Lestas, and G. Vinnicombe, "On the stability of the Foschini-Miljanic Algorithm with Time-Delays," in IEEE Conference on Decision and Control, December 2008.

[10] B. Xu, "On delay-independent stability of large-scale systems with time delays," IEEE Transaction on Automatic Control, vol. 40, no. 5, pp. 930-933, 1995.

[11] X. Liao, X. Yang, and W. Zhang, "Delay-dependent asymptotic stability for neural networks with time-varying delays," Discrete Dynamics in Nature and Society, vol. 2006, pp. Article ID 91725, 25 pages, 2006.

[12] S. Boyd, L. El Ghaoui, E. Feron, and V. Balakrishnan, Linear Matrix Inequalities in System and Control Theory. Philadelphia, USA: SIAM, 1994, in Studies in Applied Mathematics, vol.15.

[13] B. Krishnamachari, Networking Wireless Sensors. New York, USA: Cambridge University Press, 2005.

[14] R. A. Horn and C. R. Johnson, Matrix Analysis. Cambridge University Press, 1985.

[15] - Topics in Matrix Analysis. Cambridge University Press, 1994.

[16] S. Pillai, T. Suel, and S. Cha, "The Perron-Frobenius theorem: some of its applications," Signal Processing Magazine, IEEE, vol. 22, pp. 62-75, March 2005.

[17] C. A. Desoer and Y. Yang, "On the generalized Nyquist stability criterion," IEEE Transaction on Automatic Control, vol. 25, no. 1, pp. 187-196, 1980.

[18] K. Gu, V. L. Kharitonov, and J. Chen, Stability of Time-Delay Systems. Birkhäuser Boston, 2003, control engineering. 